\title{
Microstrip antenna analysis using the method of fundamental solutions
}

\author{
Berardi Sensale-Rodriguez ${ }^{1, *, \dagger}$, Berardi Sensale ${ }^{1}$, Vitor M. A. Leitão ${ }^{2}$ and \\ Custódio Peixeiro ${ }^{2}$ \\ ${ }^{1}$ Facultad de Ingenieria, Universidad de la Republica, Julio Herrera y Reissig 565, Montevideo 11300, Uruguay \\ ${ }^{2}$ Instituto Superior Técnico, TULisbon, Av. Rovisco Pais, 1049-001 Lisboa, Portugal
}

\begin{abstract}
SUMMARY
The method of fundamental solutions (MFS) is proposed for the analysis of microstrip patch antennas of arbitrary shape. The MFS consists mainly in approximating the solution of a problem by a linear combination of known fundamental solutions associated with source points located outside the domain. The implementation of the MFS is simple and computationally efficient. Simulation results are obtained for rectangular, circular and triangular microstrip patch antennas. The resonance frequency and input impedance results are in good agreement with those published in the literature. Two prototypes have been fabricated and tested. Good agreement has been obtained between the MFS simulations and the experimental results. Copyright (C) 2008 John Wiley \& Sons, Ltd.
\end{abstract}

Received 29 January 2008; Revised 8 May 2008; Accepted 5 June 2008

KEY WORDS: microstrip antennas; method of fundamental solutions; cavity model; rectangular patch; circular patch; triangular patch

\section{INTRODUCTION}

In recent years, microstrip antennas have received much attention and have become one of the most successful topics in the telecommunications field. This success stems from well-known advantages, such as, low profile, low cost, ease of construction, conformal geometry and flexibility in terms of radiation pattern, gain and polarization. The use of microstrip antennas has been boosted by the wireless revolution of the information society technologies aiming at universal access to data and voice services. Microstrip patch antennas are used nowadays in most of the modern handsets, personal digital assistants and laptop computers.

\footnotetext{
*Correspondence to: Berardi Sensale-Rodriguez, Departamento de Electronica, Instituto de Ingenieria ElectricaFacultad de Ingenieria, Julio Herrera y Reissig 565, Montevideo 11300, Uruguay.

†E-mail: bsensale@fing.edu.uy
} 
Many methods have been proposed for the analysis of microstrip patch antennas [1,2]. They can be divided into two classes; the simple, analytical or quasi-analytical methods and the full-wave complex and computationally intense methods. The first class is very easy to implement, can provide good physical insight of the radiation mechanisms and can give general performance trends. However, they can only be applied to canonical shapes and, for a general structure, they are not accurate enough to be used in a minimal iterations' efficient design procedure. Two well-known examples of this class are the transmission line model [3] and the cavity model [4]. Many complex methods have been proposed for the analysis of microstrip antennas, namely, finite difference methods, finite element methods (FEM) and method of moments (MoM) [5]. These methods are applicable to arbitrary configurations and are usually very accurate. However, they require large computational resources. The proposed method of fundamental solutions (MFS) can be classified in between the two indicated classes of methods. It is simple to implement, it can be used in the analysis of microstrip patch antennas of arbitrary geometry and can provide reasonably accurate results. The MFS has already been used to solve other types of electromagnetic problems [6-8].

In the present implementation and formulation, the MFS assumes electromagnetic fields and excitation current constant along the substrate thickness. Therefore, it can be used only for thin substrates.

\section{METHOD OF FUNDAMENTAL SOLUTIONS}

\subsection{Problem definition}

For a microstrip patch, printed on a thin substrate, lying in the $x y$-plane, and fed by a $z$-directed current, the electric field in the substrate volume, between the patch and the ground plane, can be assumed to have only the $z$-directed component and must be a solution of the scalar Helmholtz equation subject to Neumann boundary conditions [4]

$$
\left\{\begin{array}{l}
\left(\frac{\partial^{2}}{\partial x^{2}}+\frac{\partial^{2}}{\partial y^{2}}+k^{2}\right) E_{z}=\mathrm{j} \omega \mu J_{z} \text { in } \Omega \\
\frac{\partial E_{z}}{\partial n}=0 \text { in } \partial \Omega
\end{array}\right.
$$

where $k$ is the wave number, $\omega$ the angular frequency, $\mu$ the magnetic permeability of the substrate and $n$ the outward normal on the boundary of the patch. $J_{z}$ can be modelled as $J_{z}=I \delta\left(r-r_{0}\right)$ for the case of a coaxial feed probe.

\subsection{Description of the method}

The initial ideas that led to the MFS were suggested by Kupradze and Aleksidze [9] and Arantes e Oliveira [10], and the first numerical formulation of the method was presented by Mathon and Johnston [11]. An extensive survey of the MFS and related methods is presented in [12]. 
For the Helmholtz equation

$$
\left(\frac{\partial^{2}}{\partial x^{2}}+\frac{\partial^{2}}{\partial y^{2}}+k^{2}\right) E_{z}=\mathrm{j} \omega \mu I \delta\left(r-r_{0}\right)
$$

the solution:

$$
E_{z}=\frac{\omega \mu I}{4} H_{0}^{1}\left(k\left|r-r_{0}\right|\right)
$$

where $H_{0}^{1}$ is the Hankel function of first kind and order 0 , is by definition the fundamental solution, or, in another words, it is the corresponding Green's function. This solution does not satisfy the boundary conditions of problem (1).

Problem (1) can be written as

$$
\left\{\begin{array}{l}
L u=\mathrm{j} \omega \mu I \delta\left(r-r_{0}\right) \quad \text { in } \Omega \\
\frac{\partial u}{\partial n}=q=0 \quad \text { in } \partial \Omega
\end{array}\right.
$$

where the linear operator $L$ is $\nabla^{2}+k^{2}$.

The general solution of (4) can be written as

$$
u=\hat{u}+\bar{u}
$$

where

$$
\bar{u}=\frac{\omega \mu I}{4} H_{0}^{1}\left(k\left|r-r_{0}\right|\right)
$$

Now in order to find the general solution of (4) it is needed to find $\hat{u}$, which is the particular solution. Since $L u=\mathrm{j} \omega \mu I \delta\left(r-r_{0}\right)$ and $L \bar{u}=\mathrm{j} \omega \mu I \delta\left(r-r_{0}\right)$,

$$
L \hat{u}=\left(\nabla^{2}+k^{2}\right) \hat{u}=0
$$

Since $q=\bar{q}+\hat{q}=0$,

$$
\hat{q}=-\bar{q}
$$

where

$$
\bar{q}=\frac{\partial \bar{u}}{\partial n}=-\frac{\omega \mu k I}{4} H_{1}^{(1)}\left(k\left|r-r_{0}\right|\right)\left(\frac{r-r_{0}}{\left|r-r_{0}\right|} \cdot \vec{n}\right)
$$

It can be observed that if we consider $N$ points outside $\Omega$, which will be called source points, then the functions

$$
u_{j}=\frac{\mathrm{j}}{4} H_{0}^{(1)}\left(k\left|r-r_{j}\right|\right)
$$

and their correspondent normal derivatives

$$
q_{j}=-\frac{\mathrm{j} k}{4} H_{1}^{(1)}\left(k\left|r-r_{j}\right|\right)\left(\frac{r-r_{j}}{\left|r-r_{j}\right|} \cdot \vec{n}\right)
$$

satisfy (4) in $\Omega$. As shown in Figure 1, the source points have to be located outside $\Omega$.

Now the boundary conditions must be imposed. 


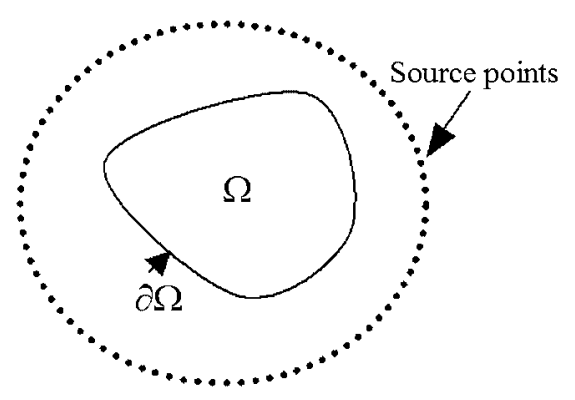

Figure 1. Geometry of the problem.

Let us write $\hat{u}$ in terms of $u_{j}$ as

$$
\hat{u}=\sum_{j=1}^{N} c_{j} u_{j}
$$

Then

$$
\hat{q}=\sum_{j=1}^{N} c_{j} q_{j}
$$

and the condition $\hat{q}=-\bar{q}$ can be written as

$$
\hat{q}=\sum_{j=1}^{N} c_{j} q_{j}=-\bar{q}=\frac{\omega \mu k I}{4} H_{1}^{(1)}\left(k\left|r-r_{0}\right|\right)\left(\frac{r-r_{0}}{\left|r-r_{0}\right|} \cdot \vec{n}\right)
$$

By applying the last equation to $M$ collocation points in the boundary

$$
\begin{aligned}
& \hat{q}\left(r_{1}\right)=\sum_{j=1}^{N} c_{j} q_{j}\left(r_{1}\right)=\frac{\omega \mu k I}{4} H_{1}^{(1)}\left(k\left|r_{1}-r_{0}\right|\right)\left(\frac{r_{1}-r_{0}}{\left|r_{1}-r_{0}\right|} \cdot \vec{n}_{1}\right) \\
& \vdots \quad \vdots \quad \vdots \\
& \hat{q}\left(r_{M}\right)=\sum_{j=1}^{N} c_{j} q_{j}\left(r_{N}\right)=\frac{\omega \mu k I}{4} H_{1}^{(1)}\left(k\left|r_{M}-r_{0}\right|\right)\left(\frac{r_{M}-r_{0}}{\left|r_{M}-r_{0}\right|} \cdot \vec{n}_{M}\right)
\end{aligned}
$$

the following linear system of equations is obtained:

$$
\left(\begin{array}{cccc}
q_{1}\left(r_{1}\right) & q_{2}\left(r_{1}\right) & \cdots & q_{N}\left(r_{1}\right) \\
q_{1}\left(r_{2}\right) & q_{2}\left(r_{2}\right) & \cdots & q_{N}\left(r_{2}\right) \\
\vdots & \vdots & \ddots & \vdots \\
q_{1}\left(r_{M}\right) & q_{2}\left(r_{M}\right) & \cdots & q_{N}\left(r_{M}\right)
\end{array}\right)\left(\begin{array}{c}
c_{1} \\
c_{2} \\
\vdots \\
c_{N}
\end{array}\right)=\left(\begin{array}{c}
-\bar{q}\left(r_{1}\right) \\
-\bar{q}\left(r_{2}\right) \\
\vdots \\
-\bar{q}\left(r_{M}\right)
\end{array}\right)
$$

By solving (16), the coefficients $c_{j}$ are obtained. Then $\hat{u}$ can be calculated and so $u$, the solution of (4), which represents the $z$-component of the electric field in the substrate under the patch. 
The source point locations depend on the specific configuration of $\Omega$ but they must necessarily be outside $\Omega$.

\subsection{Numerical convergence of the method}

The accuracy of the MFS results depends on both the number of collocation points in the boundary $(M)$ and the number of fundamental solutions $(N)$. As shown in Figure 2, for a typical rectangular patch $\left(\varepsilon_{\mathrm{r}}=2.62, \tan \delta=0.001, h=1.6 \mathrm{~mm}, L=76 \mathrm{~mm}, W=114 \mathrm{~mm}, d=23 \mathrm{~mm}\right)$, both the resonance frequency and the input resonance resistance converge quickly.

The convergence of the results was studied for values of $N / M=\left\{\frac{1}{4}, \frac{1}{2}, \frac{3}{4}, 1\right.$ and 2$\}$ showing in every case the same rate of convergence as it can be seen in Figure 2.

For regular shapes, as in Figure 2, the best convergence is obtained for values of $N / M$ greater than $\frac{1}{2}$.
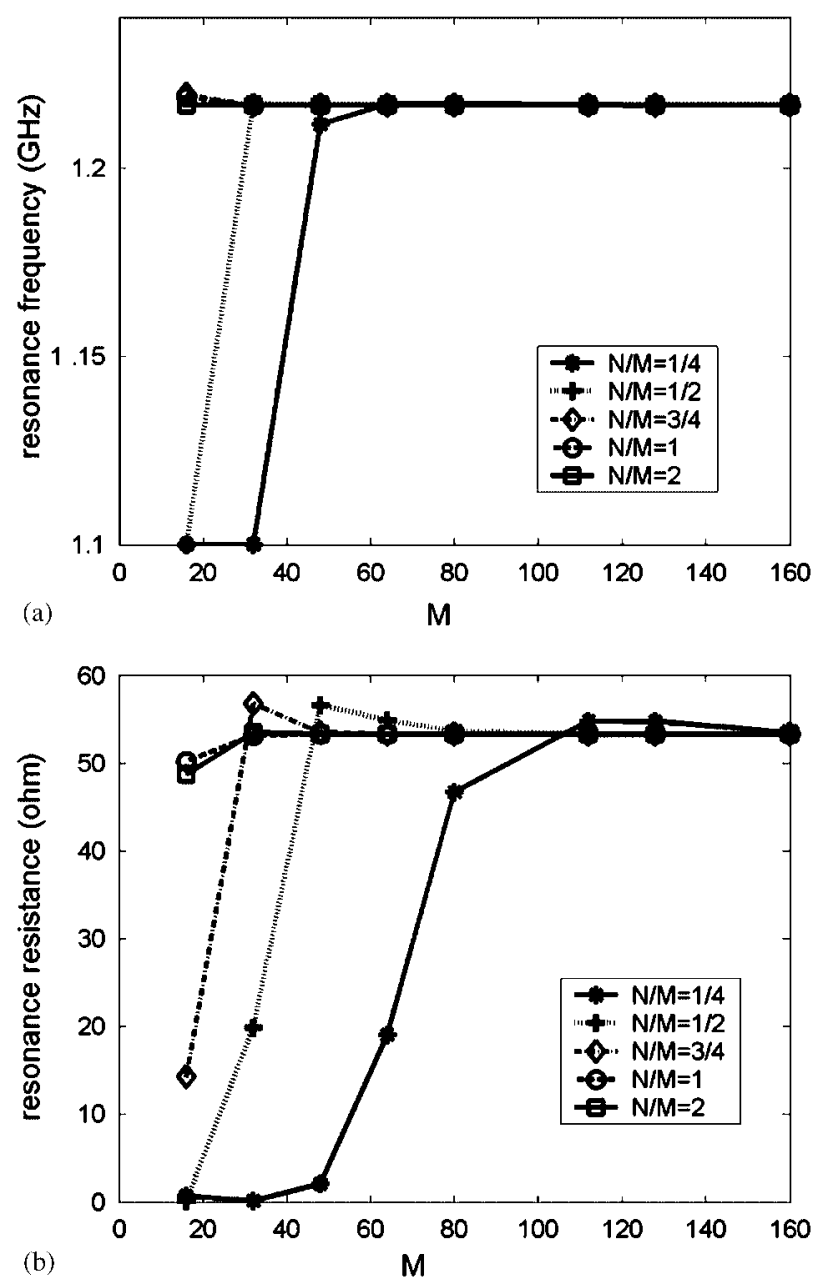

Figure 2. Convergence of MFS results: (a) resonance frequency and (b) resonance resistance. 
The percentage error (relative to the final converged result) for the resonance frequency for values of $N / M$ larger than $\frac{1}{2}$ and $M=40$ is very small (in the order of $0.005 \%$ ). For the input resonance resistance, it can be seen from Figure 2 that for a fixed $M$ the convergence is faster by increasing $N$, for values of $N / M$ larger than $\frac{3}{4}$ and $M=40$ the percentage error is less than $0.2 \%$. The evolution of the computation time used to obtain the results shown in Figure 2 is shown in Figure 3. A PC with a Pentium 3 processor at $800 \mathrm{MHz}$ and $256 \mathrm{MB}$ of RAM has been used. A good accuracy has been reached in less than $10 \mathrm{~s}$.

As it could be expected, Figure 4 shows that by choosing a lower $N / M$ ratio (for a fixed $M$ ) the computation time decreases. However, the accuracy also decreases slightly, but it remains

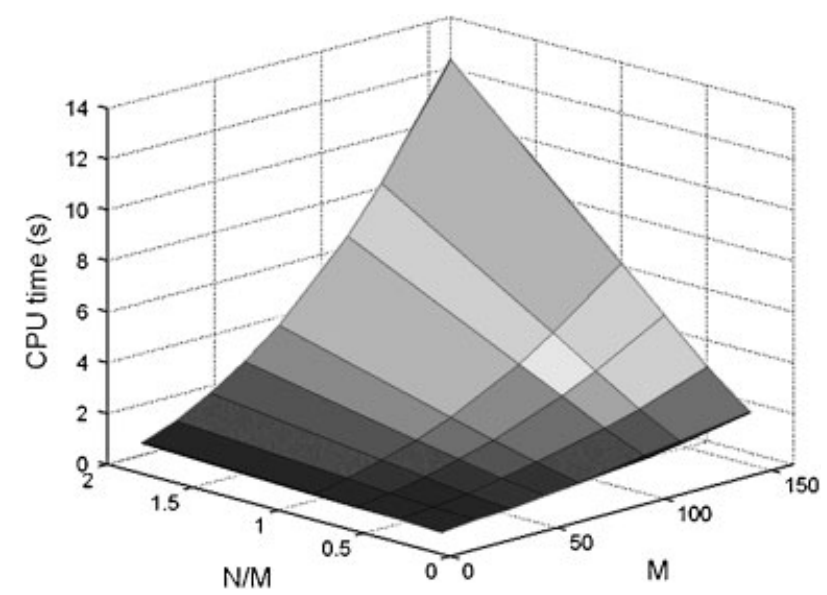

Figure 3. Computation time as a function of $M$ and $N / M$.

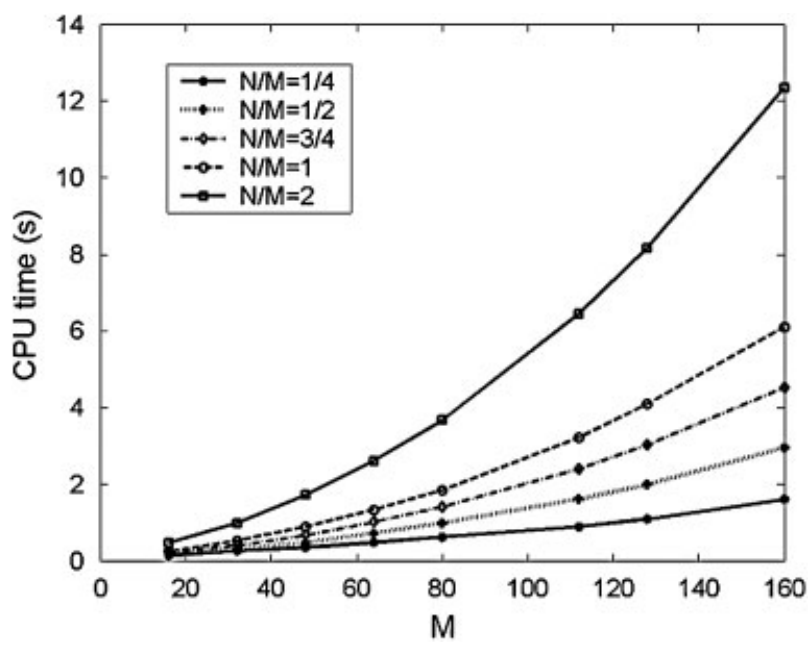

Figure 4. Computation time as a function of $N / M$. 
acceptable for $M>40$. As in other applications of the MFS [13], it was verified that, for regular shapes, the $N$ source points could be located on almost any curve outside the patch surface $(\Omega)$. However, if this source points curve is close to the patch boundary $(\partial \Omega)$ the accuracy decreases.

\subsection{Numerical stability of the method}

As in all collocation procedures, the system of equations is more likely to show deficient numerical conditioning than in weighted residuals based techniques (such as FEM).

In the context of the MFS, the condition number of the system of equations is strongly influenced by the following aspects: the number of sources, the position of the sources (the distances to the boundary) and the number and position of the collocation points.

The effect of each of these factors on the condition number of the resulting system is illustrated in Figure 5 (for the case of a rectangular domain): first, by keeping a fixed number of sources and collocation points and by varying the distance of the sources to the boundary; then, by keeping the distance and the number of sources fixed and varying the number of collocation points and, finally, by keeping the distance and number of collocation points fixed and varying the number of sources. In all cases, it is possible to see that the condition number grows (with the increase in the number of equations and/or unknowns) up to a certain (high) value and then stabilizes.

In general, numerical conditioning, on its own, may not be an unsurpassable barrier, that is, a high condition number might not necessarily mean loss of stability or accuracy of the system.

There are ways of dealing with high condition numbers either by means of optimization (trying to optimize the position and number of the sources and collocation points) or by using appropriate solvers that avoid inverting the matrices. One such technique that does not rely on the straightforward inverse of the matrix but rather uses a pseudo-inverse thus allowing for good results even for large systems of equations, is the Moore-Penrose pseudo-inverse solver [14] that is based on the singular value decomposition, which is the one used in this work.

\subsection{Implementation of the method}

The method has been implemented in Matlab, using version 6.5. The antenna geometry can be input in, basically, two ways: (1) by giving the location of the MFS source and collocation points; (2) by simply giving the equation of the curves that define $\partial \Omega$ and specifying the number of boundary points in them. If $\partial \Omega$ is made of $N$ straight segments, another option is to enter the location of the initial and final points of each segment and the number of points per segment.

The substrate parameters are simply entered as input variables of the program. So, the input variables are the coordinates of the MFS source points and collocation points, the coaxial probe location, the substrate parameters and the frequency.

As indicated previously, the MFS source points can be located on almost any curve outside $\Omega$, so they were located in a homothetic curve [15], usually of ratio 2 .

To calculate the effective loss factor, it is necessary to obtain the energy stored in the cavity and the radiated power. This calculation is done by numerical integration of the electromagnetic field.

The output data is, for a given frequency, the input impedance at that frequency. The program can plot, for an interval of frequencies, the input resistance and reactance, and the input reflection coefficient. It can also calculate the resonance frequencies and resistances, simply by using a minimization algorithm. 

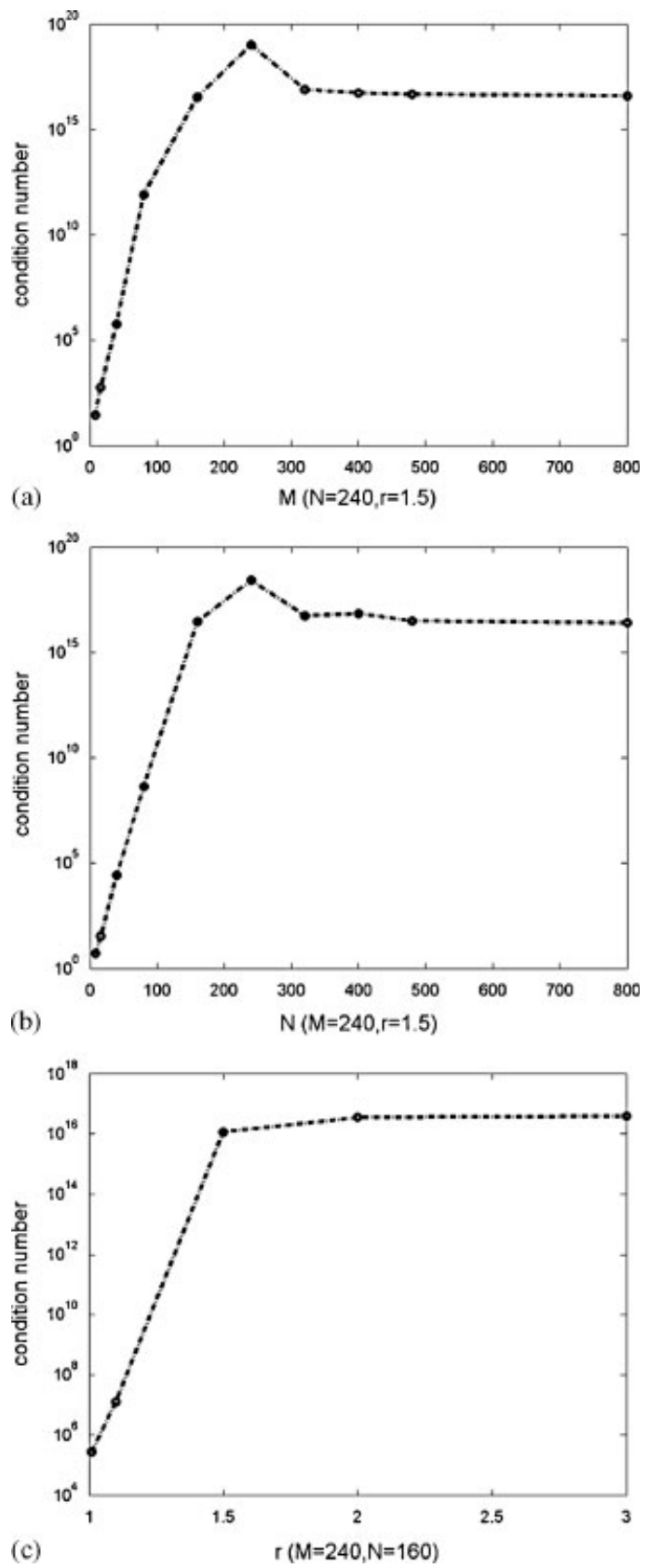

Figure 5. Condition number: (a) varying $M$; (b) varying $N$; and (c) varying distance of the sources $(r)$. 


\section{CALCULATION OF ANTENNA INPUT PARAMETERS}

To take into account the fringing field in the case of regular shape antennas, there are empirical formulas to calculate the effective dimensions of the antenna [16]. For patches of irregular shape, the effective dimensions can be approximated using the formulas for rectangular patches [17]. The input impedance of the antenna can be computed as

$$
Z_{i}=-h E_{z}\left(r_{0}\right) / I
$$

where $h$ is the substrate thickness, $E_{z}\left(r_{0}\right)$ is the electric field in the feeding point and $I$ the injected current. $E_{z}\left(r_{0}\right)$ is evaluated using a modified value of $k, k_{\text {eff }}$ that is calculated by determining the radiation loss, dielectric loss and copper loss [18].

$$
k_{\mathrm{eff}}=k\left[\epsilon_{r}\left(1-\mathrm{j} \operatorname{tg} \delta_{\mathrm{eff}}\right)\right]^{1 / 2}
$$

The effective loss tangent is

$$
\operatorname{tg} \delta_{\mathrm{eff}}=\frac{P}{2 \omega W_{\mathrm{e}}}
$$

where $W_{\mathrm{e}}$ is the time-average power stored in the electric field under the patch, and

$$
P=P_{\mathrm{r}}+P_{\mathrm{d}}+P_{\mathrm{c}}+P_{\mathrm{s}}
$$

is the total power loss. $P_{\mathrm{r}}$ is the radiated power, $P_{\mathrm{d}}$ is the power dissipated in the dielectric, $P_{\mathrm{c}}$ is the power dissipated in the metallic walls and $P_{\mathrm{s}}$ is the power lost to surface waves. For thin dielectrics $P_{\mathrm{s}}$ can be neglected.

$W_{\text {e }}$ can be calculated numerically as

$$
W_{\mathrm{e}}=\frac{\epsilon h}{4} \int_{\Omega}|E|^{2} \mathrm{~d} s
$$

Treating the patch as an equivalent source antenna with magnetic current only along its periphery, the electric potential in the far field region is

$$
F(r)=\frac{\epsilon_{0}}{4 \pi} \int_{\partial \Omega} \frac{M\left(r^{\prime}\right) \mathrm{e}^{-\mathrm{j} k\left|r-r^{\prime}\right|}}{\left|r-r^{\prime}\right|} \mathrm{d} s^{\prime}
$$

where

$$
M=2 E_{z} \widehat{z} \times n
$$

The electric field components are calculated from

$$
\begin{aligned}
& E_{\theta}=-\mathrm{j} k\left(-\sin \phi F_{x}+\cos \phi F_{y}\right) \\
& E_{\phi}=\mathrm{j} k \cos \theta\left(\cos \phi F_{x}+\sin \phi F_{y}\right)
\end{aligned}
$$

and

$$
P_{r}=\frac{1}{2 Z} \int_{0}^{2 \pi} \int_{0}^{\pi / 2} r^{2}\left(\left|E_{\theta}\right|^{2}+\left|E_{\phi}\right|^{2}\right) \sin \theta \mathrm{d} \theta \mathrm{d} \phi
$$

where $Z$ is the air characteristic impedance.

For $P_{\mathrm{c}}$, the following expression can be adopted:

$$
P_{\mathrm{c}}=2 \frac{\Re}{2} \int_{\Omega}|H|^{2} \mathrm{~d} s
$$


where

$$
\Re=(\mu \omega / 2 \sigma)^{1 / 2}
$$

is the skin-effect surface resistance of the conductor with conductivity $\sigma$ and permeability $\mu$.

Since $W_{\mathrm{e}}=W_{\mathrm{m}}$ :

$$
\int_{\Omega}|H|^{2} \mathrm{~d} s=\frac{4 W_{\mathrm{e}}}{\mu h}
$$

And finally:

$$
P_{\mathrm{d}}=\frac{\omega \epsilon^{\prime} h}{2} \int_{\Omega}|E|^{2} \mathrm{~d} s=2 \omega W_{\mathrm{e}} \operatorname{tg} \delta
$$

The resonance frequency can be found as the frequency for which the maximum of the input resistance occurs. This can be easily determined computationally due to the fast convergence of the implemented MFS. For lossless cases, the resonant frequency can be calculated simply by studying the eigenvalues of the matrix of the system in Equation (16) [8].

\section{SIMULATION RESULTS}

The MFS has been used to find the resonance frequency and input impedance of several microstrip antennas of different shapes and parameters. For the sake of comparison and validation, the analyzed examples have been taken from the literature. The results obtained from the MFS, combined with formulas for the effective dimensions of the antennas, are in good agreement with the results published in the literature. It is important to notice that for all the cases presented, an analytical solution exists for the electric field in the substrate under the patch, and the MFS results have errors of less than 5\% when 40 points in the boundary are used. The errors go down to less than $2 \%$ by doubling the number of boundary points. For such accuracy, this method is computationally more efficient than other methods.

For the rectangular patch, case 1, included in Table I, the MFS simulated electric field in the substrate under the patch and the error relative to the analytical solution are shown in Figure 6. In this example, 80 source points and 80 boundary points have been used. The relative error is less than $1 \%$.

Figure 7 shows the calculation time required to reach a specified accuracy. As it can be seen, the MFS is several orders of magnitude faster than FEM (used, for instance, in HFSS). MFS is faster mainly because it does not have to take into account interior points to the boundary. Moreover, since MFS does not require numerical integration over the boundary elements, it is also much faster than boundary element methods (BEM).

Table I. Rectangular patch parameters.

\begin{tabular}{lllcccc}
\hline Case & $\varepsilon_{\mathrm{r}}$ & $\tan \delta$ & $h(\mathrm{~mm})$ & $L(\mathrm{~mm})$ & $W(\mathrm{~mm})$ & $d(\mathrm{~mm})$ \\
\hline 1 & 10.2 & 0.002 & 1.27 & 20.0 & 30.0 & 6.5 \\
2 & & & 2.54 & 19.0 & 30.0 & 6.5 \\
3 & 2.22 & 0.002 & 0.79 & 25.0 & 40.0 & 4.0 \\
4 & & & 0.79 & 12.5 & 20.0 & 2.0 \\
\hline
\end{tabular}



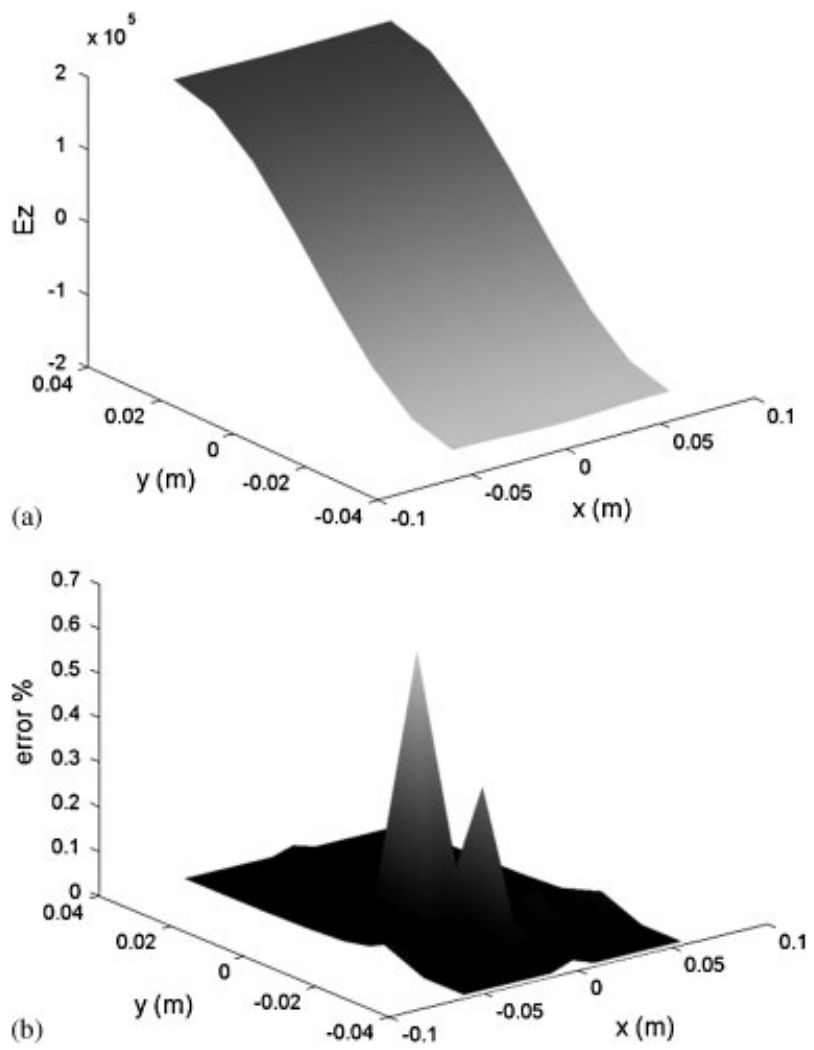

Figure 6. MFS solution for a rectangular patch: (a) electric field under the patch and (b) error relative to the analytical solution.

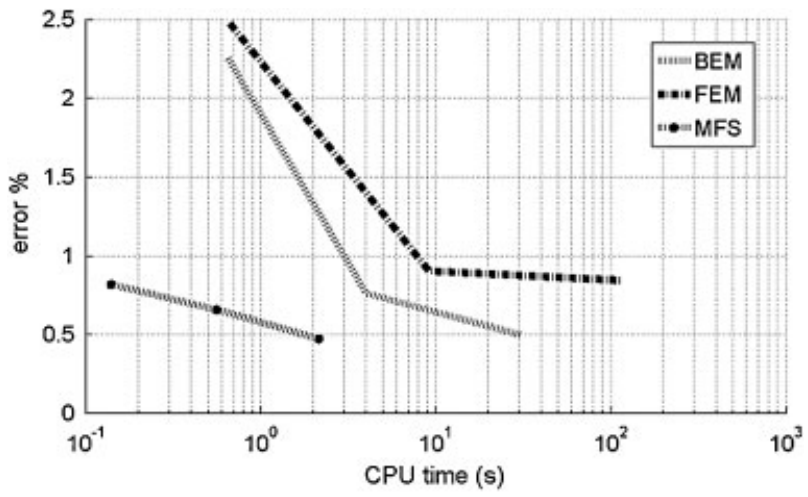

Figure 7. Comparison of computation time.

\subsection{Rectangular patch}

A generic rectangular patch geometry is shown in Figure 8. The four different rectangular patch cases indicated in Table I have been analyzed with the MFS. 
The MFS resonance frequency results are compared with other theoretical and experimental results in Table II. The MFS resonance resistance results are compared with other theoretical and experimental results in Table III. As can be concluded, very good agreement is observed between the MFS results and the measured results.

\subsection{Circular patch}

A generic circular patch geometry is shown in Figure 9. The three different circular patch cases indicated in Table IV have been analyzed with the MFS.

The MFS resonance frequency results are compared with other theoretical and experimental results in Table V. As can be observed, the MFS results and the measured results are almost identical.

\subsection{Triangular patch}

A generic equilateral triangular patch geometry is shown in Figure 10. The three different triangular patch cases indicated in Table VI have been analyzed with the MFS.

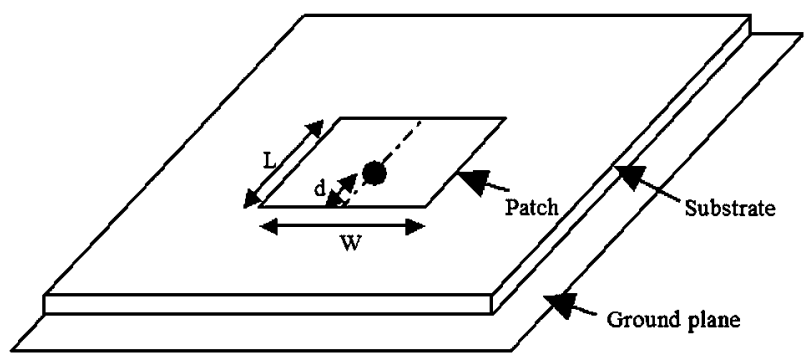

Figure 8. Rectangular patch geometry.

Table II. Rectangular patch resonance frequency results $(\mathrm{GHz})$.

\begin{tabular}{lcccc}
\hline Case & Cavity [19] & MoM [20] & Measured [19] & MFS \\
\hline 1 & 2.23 & 2.28 & 2.26 & 2.27 \\
2 & 2.22 & 2.29 & 2.24 & 2.31 \\
3 & 3.90 & 3.89 & 3.94 & 3.96 \\
4 & 7.53 & 7.61 & 7.65 & 7.79 \\
\hline
\end{tabular}

Table III. Rectangular patch resonance resistance results $(\Omega)$.

\begin{tabular}{lcccr}
\hline Case & Cavity [19] & MoM [20] & Measured [19] & MFS \\
\hline 1 & 72 & 100 & 85 & 82 \\
2 & 53 & 75 & 80 & 75 \\
3 & 83 & 101 & 89 & 98 \\
4 & 81 & 130 & 99 & 115 \\
\hline
\end{tabular}




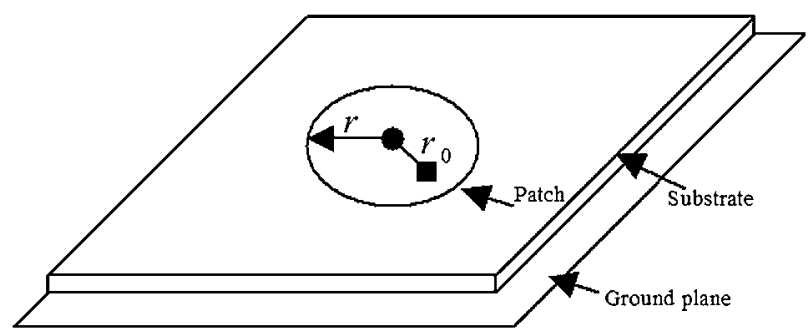

Figure 9. Circular patch geometry.

Table IV. Circular patch parameters.

\begin{tabular}{lllcrr}
\hline Case & $\varepsilon_{\mathrm{r}}$ & $\tan \delta$ & $h(\mathrm{~mm})$ & $r(\mathrm{~mm})$ & $r_{0}(\mathrm{~mm})$ \\
\hline 1 & 10.2 & 0.002 & 2.54 & 9.920 & 2.160 \\
2 & 2.33 & 0.001 & 0.79 & 14.845 & 4.150 \\
3 & 2.20 & 0.001 & 0.79 & 7.502 & 2.268 \\
\hline
\end{tabular}

Table V. Circular patch resonance frequency results $(\mathrm{GHz})$.

\begin{tabular}{lcccc}
\hline Case & Cavity [19] & MoM [20] & Measured [19] & MFS \\
\hline 1 & 2.68 & 2.50 & 2.71 & 2.70 \\
2 & 3.80 & 3.81 & 3.72 & 3.73 \\
3 & 7.73 & 7.60 & 7.38 & 7.38 \\
\hline
\end{tabular}

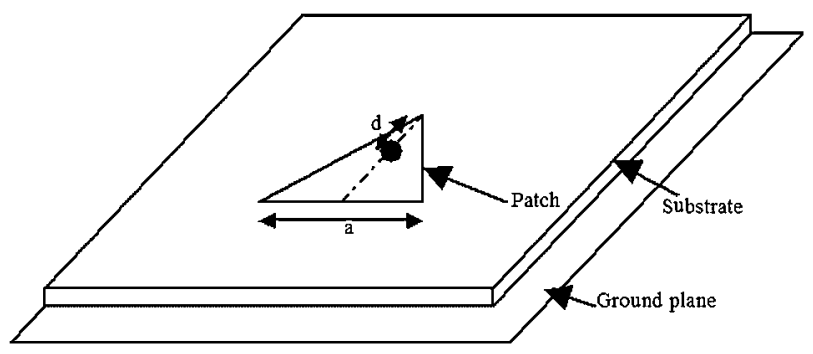

Figure 10. Equilateral triangular patch geometry.

Table VI. Triangular patch parameters.

\begin{tabular}{lcccc}
\hline Case & $\varepsilon_{\mathrm{r}}$ & $h(\mathrm{~mm})$ & $a(\mathrm{~mm})$ & $d(\mathrm{~mm})$ \\
\hline 1 & 2.32 & 1.59 & 100 & 3 \\
2 & & 0.78 & 87 & 5 \\
3 & 10.5 & 0.70 & 41 & 5 \\
\hline
\end{tabular}


Table VII. Triangular patch resonance frequency results (GHz).

\begin{tabular}{lccc}
\hline Case & MoM [21] & Measured [21] & MFS \\
\hline 1 & 1.29 & 1.28 & 1.30 \\
2 & 1.50 & 1.49 & 1.49 \\
3 & 1.52 & 1.52 & 1.50 \\
\hline
\end{tabular}

(a)

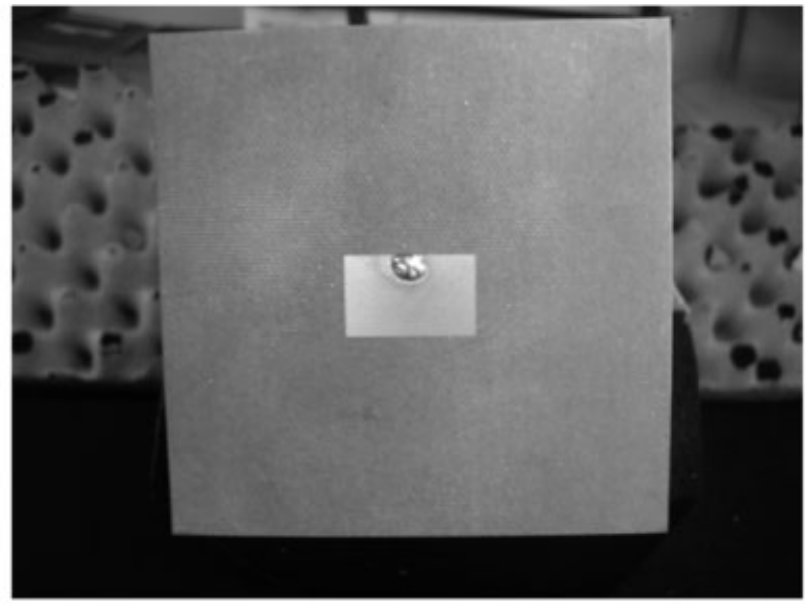

(b)

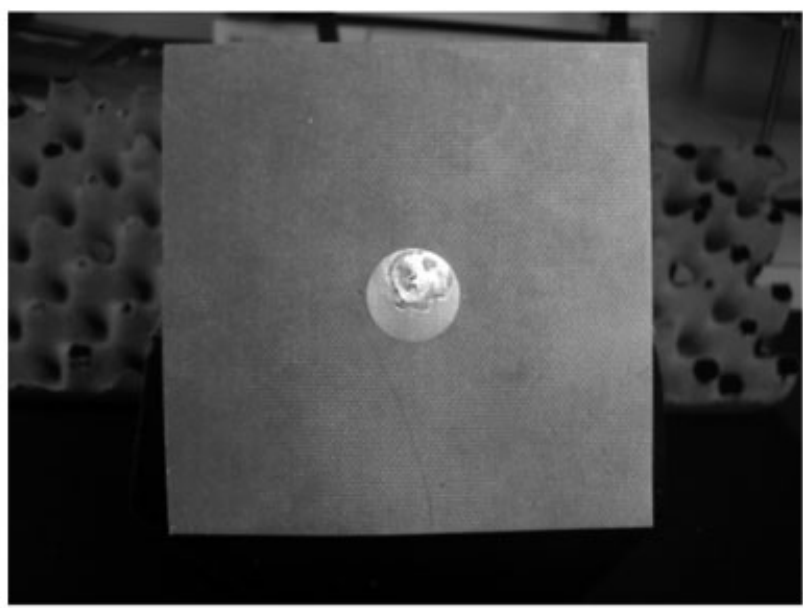

Figure 11. Microstrip patch antenna prototypes: (a) rectangular patch; and (b) circular patch.

The MFS resonance frequency results are compared with MoM and experimental results [21] in Table VII. There is once more an excellent agreement between the MFS results and the measured results. 


\section{COMPARISON WITH EXPERIMENTAL RESULTS}

Two of the analyzed microstrip patch antennas have been fabricated using conventional photolithography technique. The prototypes are shown in Figure 11. They correspond to the rectangular patch referenced as 'case 4' and the circular patch labeled as 'case 3'.

The two patches have also been analyzed with the ENSEMBLE software [22]. ENSEMBLE uses a full-wave MoM solution of the mixed-potential integral equation [23].

The input reflection coefficient of the two antenna prototypes has been measured with a network analyzer. The corresponding rectangular patch and circular patch results are compared with ENSEMBLE and MFS simulation results in Figures 12 and 13, respectively. The corresponding input impedance (resistance and reactance) results are compared in Figures 14 and 15.

Both the input reflection coefficient and the input impedance (resistance and reactance) MFS results show a relatively good agreement with experimental results. The accuracy is comparable to ENSEMBLE results. For the circular patch example (Figures 13 and 15), the agreement between MFS and experimental results is even better than for ENSEMBLE. The difference in the input reflection coefficient is about $1.5 \%$ for the MFS and $2.9 \%$ for ENSEMBLE.

\section{CONCLUSION}

The MFS has been proposed for the analysis of microstrip patch antennas. It is a very simple method based on the linear combination of known fundamental solutions of the Helmholtz equation. It provides accurate results with very scarce computation resources and can be applied to microstrip patches of arbitrary geometry. Resonance frequency results have been obtained for rectangular, circular and triangular patches. An accuracy better than $2 \%$, similar to the MoM, a much more complex and computationally demanding method, has been obtained. Extra validation has been provided by experimental results.

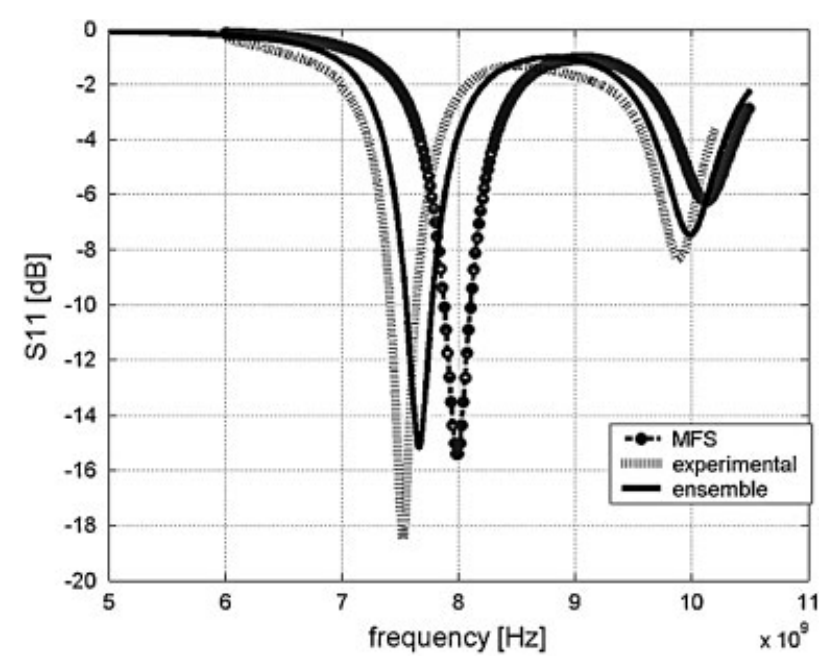

Figure 12. Comparison of simulation and experimental input reflection coefficient results of the rectangular patch. 


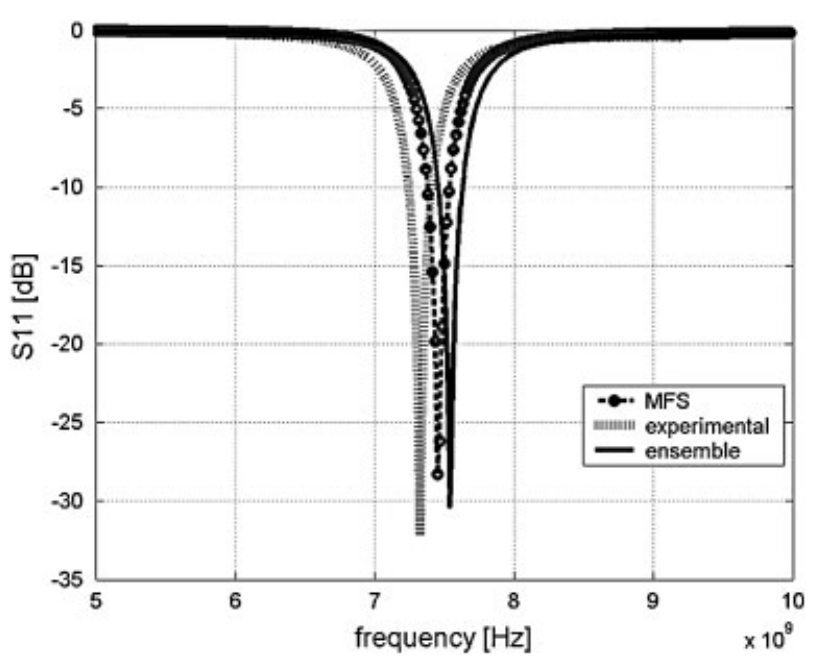

Figure 13. Comparison of simulation and experimental input reflection coefficient results of the circular patch.
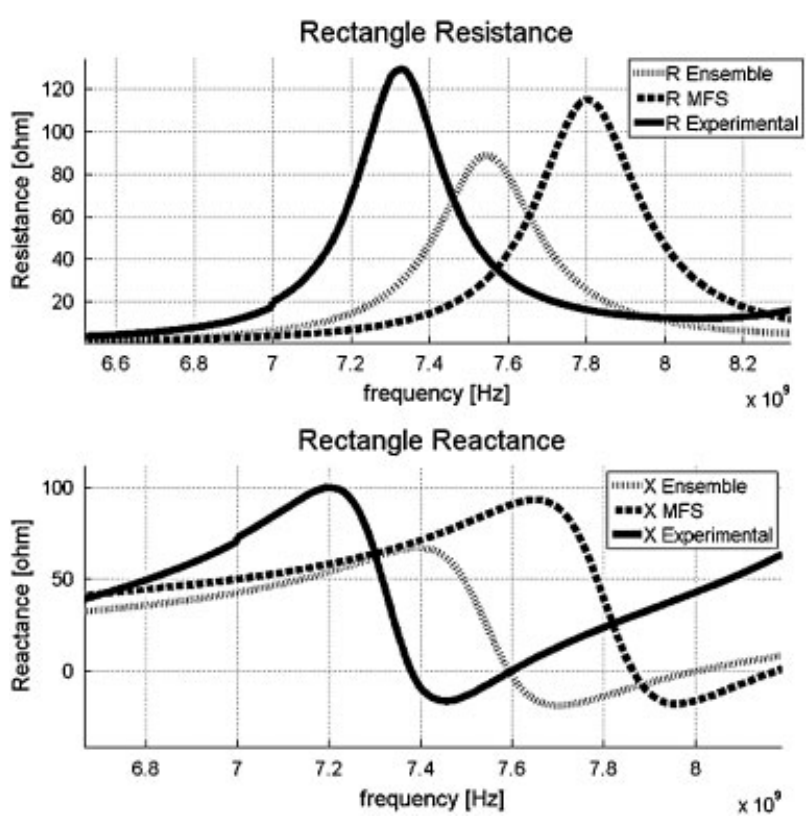

Figure 14. Comparison of simulation and experimental input impedance results of the rectangular patch.

In the present implementation, only resonance frequency and input impedance can be calculated. However, it is possible to extend the formulation to the calculation of the radiation pattern and associated antenna parameters. 

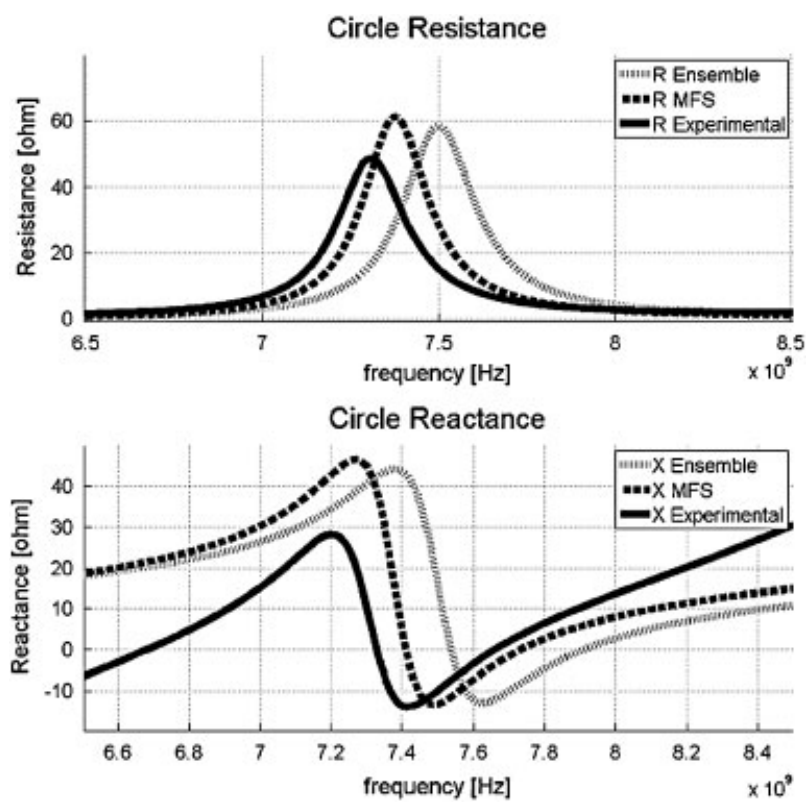

Figure 15. Comparison of simulation and experimental input impedance results of the circular patch.

\section{ACKNOWLEDGEMENTS}

The authors thank Carlos Brito and António Almeida from Instituto Superior Técnico for, respectively, the fabrication and the testing of the antenna prototypes.

\section{REFERENCES}

1. Pozar DM, Schaubert DH. Microstrip Antennas: The Analysis and Design of Microstrip Antennas and Arrays. IEEE Press: New York, 1995.

2. Garg R, Bhartia P, Bahl I, Ittipiboon A. Microstrip Antenna Design Handbook. Artech House: Boston, 2001.

3. Carver KR, Mink JW. Microstrip antenna technology. IEEE Transactions on Antennas and Propagation 1981; 29(1):2-24.

4. Lo YT, Solomon D, Richards WF. Theory and experiment on microstrip antennas. IEEE Transactions on Antennas and Propagation 1979; 27(3):137-145.

5. Hoorfar A, Jamnejad V. Electromagnetic modeling and analysis of wireless communication antennas. IEEE Microwave Magazine 2003; 4(3):51-67.

6. Karageorghis A. The method of fundamental solutions for the calculation of the eigenvalues of the Helmholtz equation. Applied Mathematics Letters 2001; 14:837-842.

7. Smyrlis YS, Karageorghis A. Some aspects of the method of fundamental solutions for certain harmonic problems. Journal of Scientific Computing 2001; 16(9):341-371.

8. Young DL, Hu SP, Chen CW, Fan CM, Murugesan K. Analysis of elliptical waveguides by the method of fundamental solutions. Microwave and Optical Technology Letters 2005; 44(3):552-558.

9. Kupradze VD, Aleksidze MA. The method of functional equations for the approximate solution of certain boundary value problems. Computational Methods in Mathematical Physics 1964; 4:82-126.

10. Arantes e Oliveira ER. Plane stress analysis by a general integral method. ASCE Engineering Mechanics Division 1968; 94(EM1):79-101.

11. Mathon R, Johnston RL. The approximate solution of elliptic boundary-value problems by fundamental solutions. SIAM Journal on Numerical Analysis 1977; 12:638-650. 
12. Fairweather G, Karageorghis A. The method of fundamental solutions for elliptic boundary value problems. Advances in Computational Mathematics 1998; 9:69-95.

13. Fam GS, Rashed YF. A study on the source points locations in the method of fundamental solutions. Boundary Elements XXIV 2002; 18:297-312.

14. Penrose R. A generalized inverse for matrices. Proceedings of the Cambridge Philosophical Society 1955; 51:406-413.

15. Gorzelánczyk P, Kolodziej JA. Some remarks concerning the shape of the source contour with application of the method of fundamental solutions to elastic torsion of prismatic rods. Engineering Analysis with Boundary Elements 2008; 32:64-75.

16. James JR, Hall PS, Wood C. Microstrip Antenna Theory and Design. Peter Peregrinus: London, 1981.

17. Palamisamy V, Garg R. Analysis of arbitrarily shaped microstrip patch antennas using segmentation technique and cavity model. IEEE Transactions on Antennas and Propagation 1986; 34(10):1208-1213.

18. Jahagirdar DR, Steward RD. Faster computations for segmentation method based on cavity model. IEEE $2 n d H F$ Postgraduate Student Colloquium 1996; 58-63.

19. Gan YB, Chua CP, Li LW. An enhanced cavity model for microstrip antennas. Microwave and Optical Technology Letter 2004; 40(3):520-523.

20. Pozar DM. Input impedance and mutual coupling of rectangular microstrip antennas. IEEE Transactions on Antennas and Propagation 1982; 35(11):1191-1196.

21. Chen W, Lee KF, Dahele JS. Theoretical and experimental studies of the resonant frequencies of the equilateral triangular microstrip antenna. IEEE Transactions on Antennas and Propagation 1992; 40(10):1253-1256.

22. ENSEMBLE Design, Review, and 1D Array Synthesis, version 5.1, User's Guide, Ansoft Corporation, January 1998.

23. Mosig JR. Arbitrarily shaped microstrip structures and their analysis with a mixed potential integral equation. IEEE Transactions on Microwave Theory and Techniques 1988; 36(2):314-323.

\section{AUTHORS’ BIOGRAPHIES}

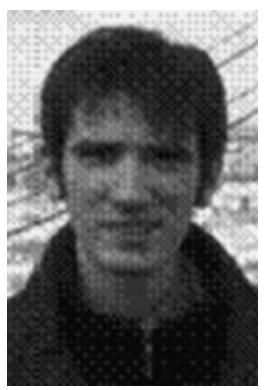

Berardi Sensale-Rodriguez was born in Montevideo, Uruguay, in 1984. He is finishing his last year project in Electrical Engineering in the Facultad de Ingenieria, Universidad de La República. He has been teaching in the Institute of Mathematics since 2005, and since 2007 in the Microelectronics group of the Department of Electronics where he is a teaching assistant and is currently working in several research projects.

His present research interests are focused on the application of numerical techniques to various problems of electronics design.

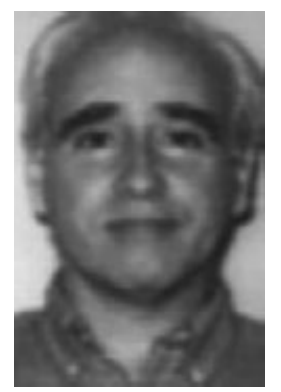

Berardi Sensale was born in Montevideo, Uruguay, in 1953. He received the graduation degree in civil and structural engineering from Facultad de Ingenieria, Universidad de la Republica, in 1980 and master and doctor degrees form Universidade Federal de Rio Grande do Sul, Brazil, in 1990 and 1997, respectively. He has been teaching in the Institute of Structures and Transportation since 1985 where he is now a full professor.

His present research interest is in the investigation on mesh-free methods. 


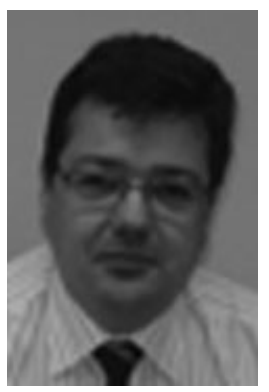

Vitor M. A. Leitão was born in Braga, Portugal, in 1964. He received the graduation and master degrees in civil and structural engineering from Instituto Superior Técnico (IST), Technical University of Lisbon, in 1987 and 1990, respectively, and a PhD from the University of Portsmouth, U.K., in 1994. He has been teaching in the Department of Civil Engineering and Architecture since 1987 where he is now an Associate Professor. He is also a researcher of ICIST-Instituto de Engenharia de Estruturas, Território e Construção. His present research interests are focused on the development of the method of fundamental solutions and other mesh-reducing techniques with applications to various engineering problems.

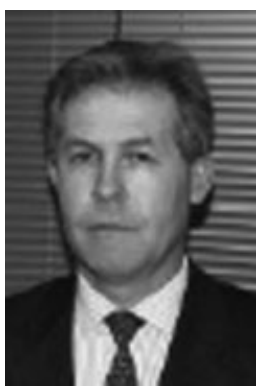

Custódio Peixeiro was born in Évora, Portugal, in 1956. He received the graduation, master and doctor degrees in electrical and computer engineering from Instituto Superior Técnico (IST), Technical University of Lisbon, in 1980, 1985 and 1993, respectively. He has been teaching in the Department of Electrical and Computer Engineering since 1980 where he is now an Assistant Professor. He is also a researcher of Instituto de Telecomunicações.

His present research interests are focused on microstrip antennas and circuits for applications in mobile terminals (handsets, PDAs and laptop computers). 